\title{
Serpents et échelles. Du jeu de hasard à l'expérience de la transcendance
}

Snakes and Ladders. From Game of Chance to the Experience of Transcendence

\section{Serge Finck et Patrick Schmoll}

\section{OpenEdition}

Journals

Édition électronique

URL : https://journals.openedition.org/sdj/3807

DOI : $10.4000 /$ sdj.3807

ISSN : 2269-2657

Éditeur

Laboratoire EXPERICE - Centre de Recherche Interuniversitaire Expérience Ressources Culturelles Education

\section{Référence électronique}

Serge Finck et Patrick Schmoll, « Serpents et échelles. Du jeu de hasard à l'expérience de la transcendance », Sciences du jeu [En ligne], 16 | 2021, mis en ligne le 14 novembre 2021, consulté le 26 novembre 2021. URL : http://journals.openedition.org/sdj/3807 ; DOI : https://doi.org/10.4000/sdj. 3807

Ce document a été généré automatiquement le 26 novembre 2021.

\section{c)}

La revue Sciences du jeu est mise à disposition selon les termes de la Licence Creative Commons Attribution - Pas d'Utilisation Commerciale - Pas de Modification 4.0 International. 


\title{
Serpents et échelles. Du jeu de hasard à l'expérience de la transcendance
}

Snakes and Ladders. From Game of Chance to the Experience of Transcendence

\author{
Serge Finck et Patrick Schmoll
}

1 Le jeu des Serpents et échelles n'est pas très connu en Europe latine, mais dans le monde anglo-américain il est pratiqué par les enfants aussi couramment que chez nous le jeu de l'Oie ou les Petits chevaux. Sans doute pour cette raison a-t-il fait l'objet d'assez peu de travaux scientifiques dans la sphère francophone, alors que plusieurs articles et ouvrages en anglais lui sont consacrés dans des domaines associant sciences du jeu, mathématiques, sciences des religions, philosophie et arts. Nous saisirons donc l'opportunité de cet article pour, au-delà de la visée particulière annoncée par son titre, dresser une présentation générale de ce jeu et un état des recherches le concernant. 
Illustration 1 : Schéma d'un plateau de Serpents et échelles à cent cases, dans une version à six serpents et six échelles (Dessin des auteurs).

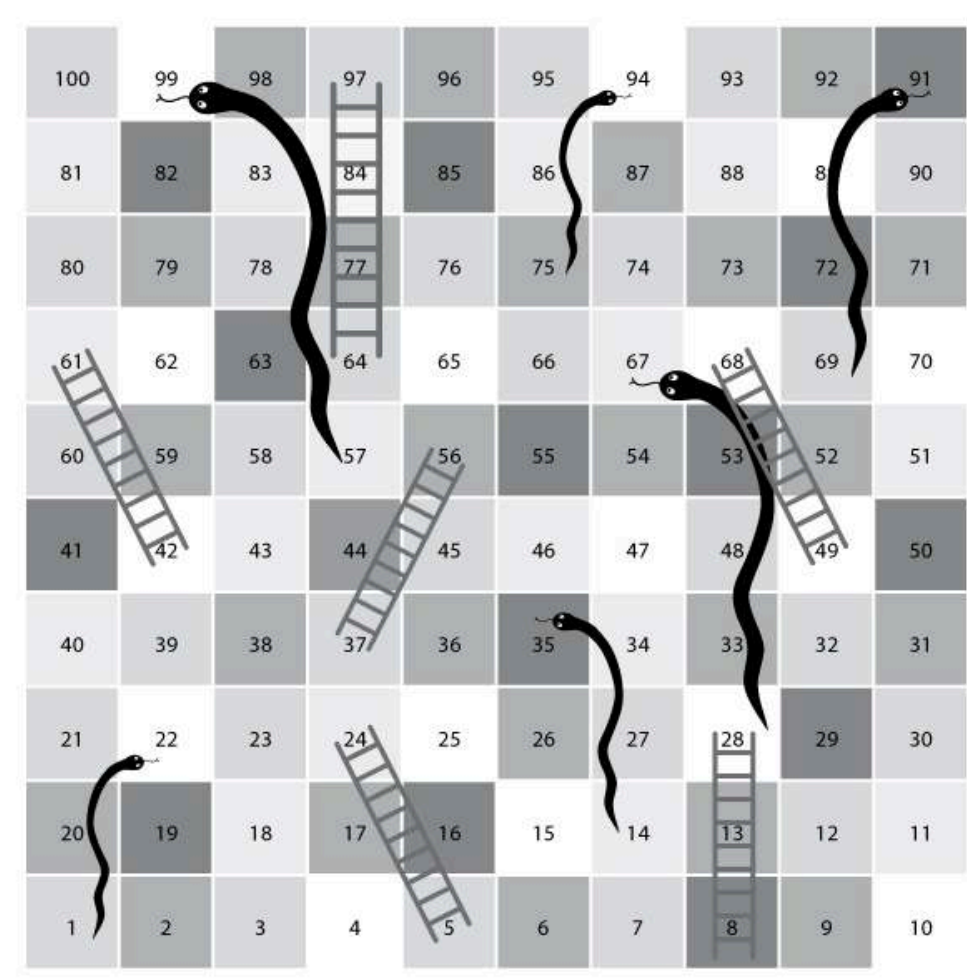

2 Notre propos traitera plus particulièrement d'un aspect de ce jeu généralement ignoré de ceux qui n'en connaissent que la version couramment diffusée sur les présentoirs de nos magasins : il s'agit de sa portée sotériologique ${ }^{1}$. Serpents et échelles est en effet la version profane, diffusée aujourd'hui en Occident, d'un jeu qui est pratiqué depuis des siècles en Inde, sous différentes versions selon les cultures, les religions et les époques, sous différents modes de jouer (divertissement, éducation morale, voire consultation oraculaire ou expérience simulée du samsara, le cycle des morts et des renaissances), ainsi que sous différentes désignations : Paramapada Sopana Patam ( «jeu de l'échelle du salut»), Moksha Patam («jeu de la libération») ou Gyan Chaupar («jeu de la connaissance »). Les travaux que nous avons consultés titrent le plus fréquemment sous cette dernière appellation de Gyan Chaupar: c'est par commodité celle que nous retiendrons ici, alternativement à celle de Serpents et échelles, quand nous nous référerons aux versions indiennes du jeu.

3 L'ancienneté du jeu, la persistance d'une structure formelle identifiable à travers ses variantes hindoues, jaïns ou bouddhistes, et jusque dans sa réception dans l'Angleterre victorienne au XIX ${ }^{e}$ siècle, mais aussi dans le monde musulman à l'époque moghole, et sa diffusion mondiale dans une version désormais exempte de contenus religieux ou moraux, amènent à se poser la question de ce qui se passe dans le transfert du jeu d'une culture à une autre. Ou plus exactement : dans la transformation en jeu d'un dispositif et d'une pratique qui, dans leur contexte culturel d'origine, ne relèvent pas clairement du jeu.

4 Le traitement de ces questions est rendu délicat par le présupposé des termes utilisés : nous aurions notamment une définition stable et transculturelle de "ce qu'est un jeu ", qui nous permettrait d'établir que les versions indiennes de Serpents et échelles, en 
raison de leur usage pédagogique, moral, divinatoire ou sotériologique, «ne sont pas des jeux ». Mais le terme chaupar, par exemple, désigne bien un jeu de dés sur plateau. Ne pourrait-on prendre le contrepied en partant de ce que leurs utilisateurs désignent comme « ceci est un jeu»? Ce qui nous permettrait d'approcher Serpents et échelles en tant que, dans ses diverses versions, il nous dit quelque chose de ce qu'est « un jeu » et « jouer » dans différentes cultures. On serait ainsi amené à interroger, pour en pointer le relativisme culturel, les catégories un peu trop fermes qui définissent canoniquement le jeu en Occident, et notamment la différence entre jeu et sacré ou la frontière tranchée entre un monde qui serait celui de la réalité quotidienne, sérieuse et tangible, et un monde fictionnel qui serait celui du jeu.

\section{Règles du jeu}

$5 \quad$ Les règles font partie des invariants qui rendent le jeu reconnaissable au-delà de ses diverses déclinaisons. Serpents et échelles utilise un plateau figurant une grille de cases numérotées, sur laquelle chaque joueur déplace son pion en fonction du résultat d'un jet de dé. De nos jours, on utilise un seul dé à six faces. Traditionnellement, en Inde, on avait recours à plusieurs dés oblongs à quatre faces (symbolisant les quatre yugas ou âges du monde) ou à des cauris, coquillages-monnaie interprétés en fonction de leur position (Van Langendonckt, 2013).

6 Le parcours du pion suit un boustrophédon : partant généralement de la case 1, en bas à gauche, il suit la première ligne de cases jusqu'au bout, puis revient en sens inverse par la ligne suivante située au-dessus. Dans certaines versions indiennes, on part d'une ligne située plus haut, les lignes laissées en dessous offrant des cases où le pion peut éventuellement rétrograder, certaines pouvant faire fonction de "prisons" ou « d'enfers » où l'on passe son tour. Le but est d'être le premier à atteindre la dernière case qui, selon les variantes du jeu, se trouve au bout de la dernière ligne ou au milieu de celle-ci.

7 Sur le plateau, des échelles et des serpents relient certaines cases. Si le pion du joueur tombe sur une case où figure le bas d'une échelle, il grimpe directement à la case au sommet de celle-ci. S'il tombe sur une case où figure la tête d'un serpent, il est avalé et retourne à l'extrémité de sa queue.

8 Le jeu connaît de nombreuses variantes de ce principe de base, selon les époques et les aires culturelles. La taille de la grille et le nombre de cases vont couramment de 64 $(8 \times 8)$ à $100(10 \times 10)$ dans les versions occidentales de Serpents et échelles. Mais de nombreuses versions existent dans des formats différents (cf. par ex. illustration 2). 
Illustration 2 : Une version particulière de Serpents et échelles réduite à 48 cases $(6 \times 8)$.

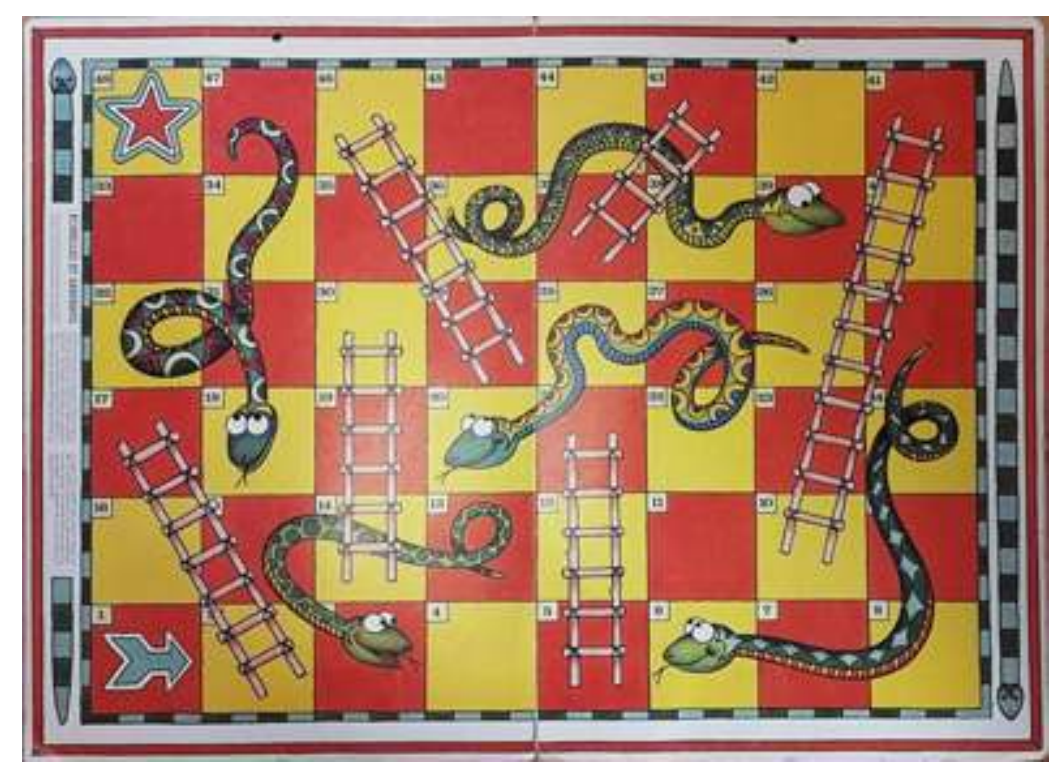

London, The Hamlyn Publishing Group, 1988. Jeu, illustrations et texte de Caroline \& John Astrop.

9 Les versions hindoues du Gyan Chaupar sont souvent des ashtapada (8 x 8) ou des dasapada (10 x 10) (Van Langendonckt 2013), mais présentent aussi des grilles allant de 72 cases $(9 \times 8)$ jusqu'à $124(12 \times 12)$, voire $140(14 \times 10)$ ou 380 (Topsfield 2006b). La version jaïn du jeu (illustrations 3 et 4 ) comporte 84 cases, distribuées sur une grille de $9 \times 9$ augmentée de trois cases en excroissance (1,56 et 66). Cinq cases supplémentaires (1 à 5), au-delà de la case terminale, représentent un séjour céleste. Une version musulmane fut utilisée en Inde pendant la période moghole, entre la fin du XVII ${ }^{\mathrm{e}}$ siècle et le début du XVIII , et comporte 100 cases représentant le nombre des noms de Dieu, ou 101 si le trône d'Allah y est figuré (Topsfield, 2006b). Le nombre des échelles et des serpents varie également, en fonction des dimensions de la grille, l'ensemble influant sur la durée de la partie. 
Illustration 3 : Gyan Chaupar, version jaïn du jeu

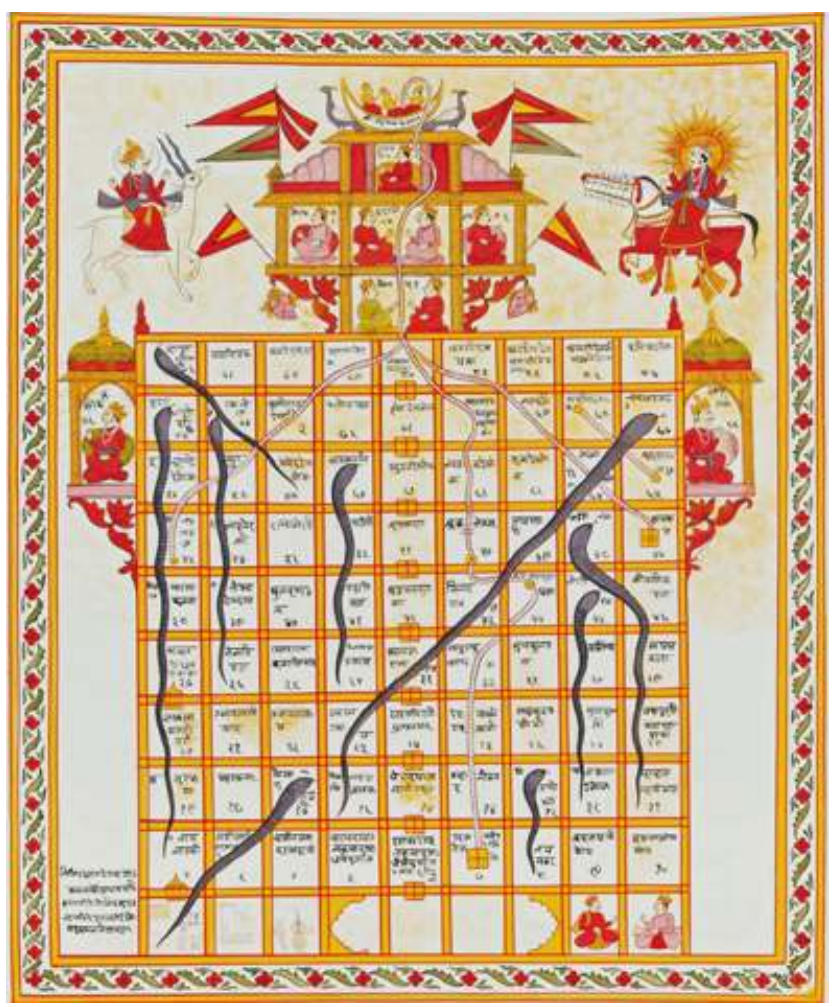

XIXe siècle, gouache sur toile, anonyme.

https://commons.wikimedia.org/wiki/File :Snakes_and_Ladders.jpg 
Illustration 4 : Gyan Chaupar, version jain du jeu

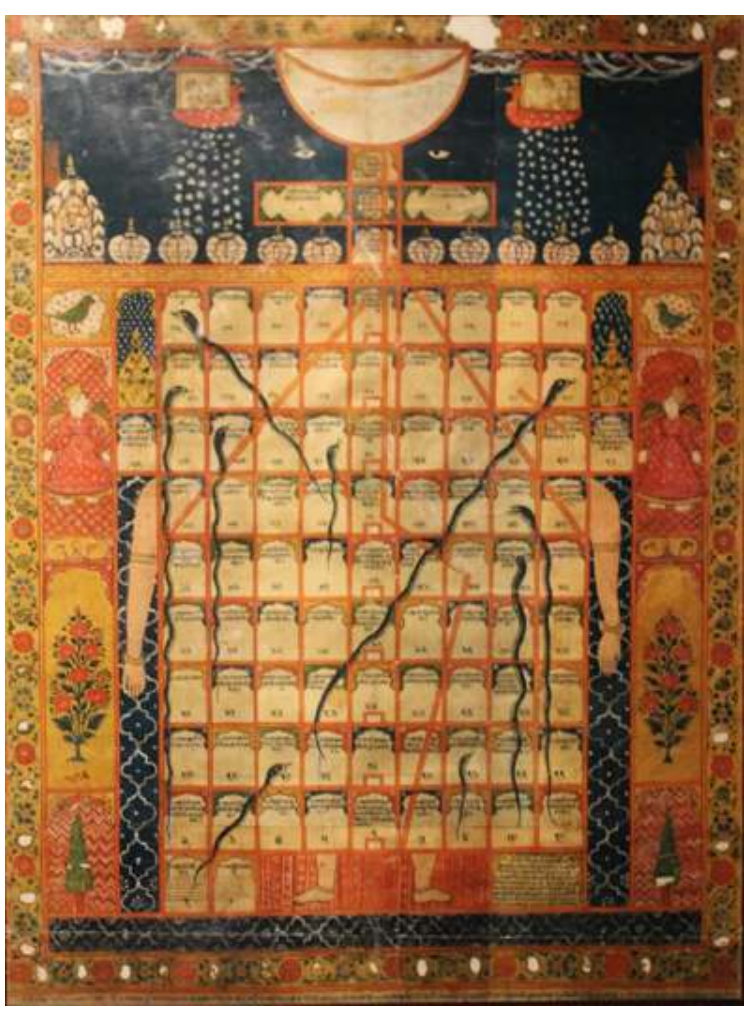

XIXe siècle, gouache sur toile, anonyme.

National Museum of India, New Delhi.

https://commons.wikimedia.org/wiki/File:Gyan_chaupar.JPG.

10 Avant de pouvoir démarrer, le joueur doit tirer un six (dans la version se jouant avec un dé cubique). Il peut alors placer son pion sur la case 1. Il relance ensuite et déplace son pion du nombre de cases correspondant à son jet. Lorsqu'il tire un six, il déplace son pion d'autant et a droit à un second jet. Dans la version proposée par Harish Johari (1993) à partir d'un exemplaire indien du jeu datant du XIX siècle, si le joueur tire un six trois fois de suite, il lui faut en tirer un quatrième pour continuer, sinon il revient à la case où il se trouvait avant sa série de six, et avance du nombre obtenu à son quatrième jet.

11 Pour gagner, en fin de parcours, il faut tirer le nombre exact permettant de tomber sur la dernière case. Si le nombre tiré est supérieur, le pion connaît différents destins selon les variantes, mais qui reviennent tous à ce qu'il rétrograde pour tenter un nouvel essai au tour suivant. Sur les grilles dont la dernière case est en bout de ligne, le joueur recule du nombre de cases correspondant à l'excédent. Sur les grilles dont la case terminale est au milieu de la dernière ligne, le joueur qui ne tombe pas précisément sur cette case poursuit son parcours et rétrograde de l'excédent. Parfois, un serpent en bout de ligne l'oblige à redescendre une ou plusieurs lignes en-dessous.

\section{Histoire du jeu}

La première édition de Serpents et échelles en Occident date du XIX ${ }^{e}$ siècle. Son origine indienne, quant à elle, remonte à plusieurs siècles, mais son ancienneté est débattue. 
Topsfield (1985) constate que très peu de plateaux de Gyan Chaupar datent d'avant le $\mathrm{XVII}{ }^{\mathrm{e}}$ siècle, et qu'aucun datant d'avant le XVI ${ }^{\mathrm{e}}$ siècle ne nous est parvenu. L'existence du jeu est cependant attestée dans la tradition à des époques plus anciennes. Sous son appellation Moksha Patam, le jeu aurait été inventé pour les enfants par un poète du $\mathrm{XIII}^{\mathrm{e}}$ siècle, Dnyaneshwar, alias Gyandev, mais nous n'avons pas pu en trouver confirmation dans les biographies accessibles du personnage. Mark Tatz et Jody Kent (1977) présentent une variante tibétaine à 72 cases $(9 \times 8)$, qui passe pour avoir été conçue par un moine érudit tibétain, Sakya Pandita (1182-1251) sur la base d'un jeu indo-népalais préexistant, nommé Nagapasa («serpents et dé», ou «pièges des serpents »). Deepak Shimkada (1983) pense que l'origine du jeu est effectivement bouddhiste, parce que les 72 cases sont cohérentes avec les significations numérologiques du bouddhisme indien. Plus récemment, l'indologue danois Jacob Schmidt-Madsen (2019) conteste l'ancienneté attribuée au jeu, dont il ne voit pas d'exemplaires indiscutables avant le XVIII ${ }^{e}$ siècle.

Le terme chaupar renvoie en effet à un jeu de hasard sur plateau, où les pièces se déplacent en fonction du résultat d'un jet de dé. Il désigne donc parfois assez indifféremment deux types de jeu : le Gyan Chaupar, qui a inspiré Serpents et échelles, et le Pachisi, qui se joue sur un tablier en forme de croix et dont sont issus, en Occident, le Ludo et les Petits chevaux (illustration 5). Il est difficile de distinguer quel type désignent les textes anciens quand ils parlent d'un jeu de dés.

Illustration 5 : tablier de Pachisi

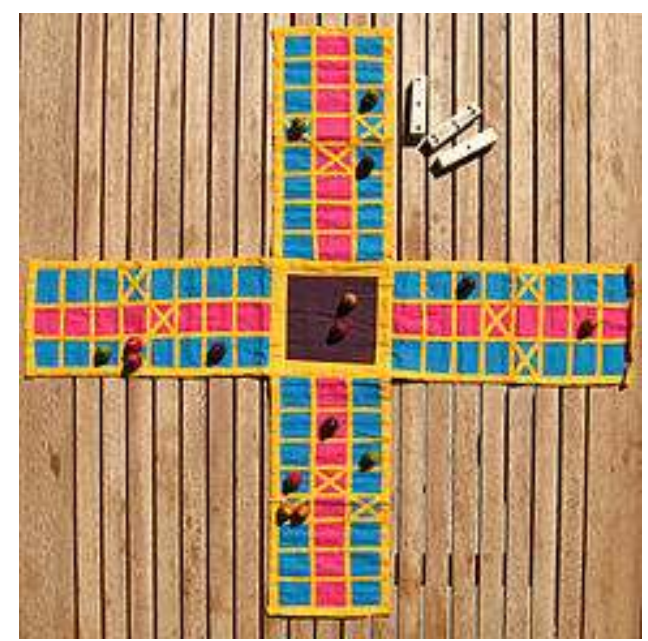

Photo : Micha L. Rieser, https://commons.wikimedia.org/wiki/File :Pachisi-real.jpg

On a retrouvé des dés en terre cuite, en os et en ivoire sur les sites archéologiques de la civilisation de la vallée de l'Indus (III ${ }^{\mathrm{e}}$ millénaire av. J.-C.), de même que des artefacts dans lesquels Andrew Topsfield (2006a) voit des plateaux de jeu. Le Rig-Veda (II ${ }^{\mathrm{e}}$ millénaire av. J.-C.) consacre l'un de ses hymnes au jeu de dés ${ }^{2}$ : le joueur y chante sa passion pour le jeu, se lamente de la perte de ses biens et de son épouse, et prie le dé de le prendre en pitié et de le libérer de son emprise magique. Le jeu de dés est également au centre d'un épisode dramatique du Mahabharata, un poème épique rédigé dans sa forme finale au IV $\mathrm{V}^{\mathrm{e}}$ siècle apr. J.-C., mais qui évoque un contexte remontant aux IX ${ }^{\mathrm{e}}$ VIII siècle av. J.-C.: Yudhishthira et les Pandavas perdent leur royaume, tous leurs 
biens, y compris eux-mêmes et leur femme Draupadi, en jouant au Pachisi avec leurs cousins. Dans la Skanda Purana (VI ${ }^{\mathrm{e}}$ siècle), Shiva et Parvati, divinités mariées, mais antagonistes, jouent le destin des hommes aux dés sur un plateau de jeu. La scène a été représentée dans de nombreuses peintures et sculptures, les montrant souvent autour d'un Pachisi (illustration 6), ou d'un plateau de jeu dont on ne peut cependant affirmer que ce soit un Gyan Chaupar (illustration 7). On trouve une grande concentration de ces scènes sculptées dans les grottes d'Ellora (centre ouest de l'Inde) (illustration 8). Micaela Soar (2006) en a analysé une vingtaine, allant $\mathrm{du} \mathrm{II}^{\mathrm{e}}$ siècle avant au $\mathrm{X}^{\mathrm{e}}$ siècle apr. J.-C., qui condensent une symbolique sexuelle et des rapports de domination et de tricherie au sein du couple qui se dispute le pouvoir sur terre.

\section{Illustration 6 : Shiva et Parvati jouant au Pachisi}

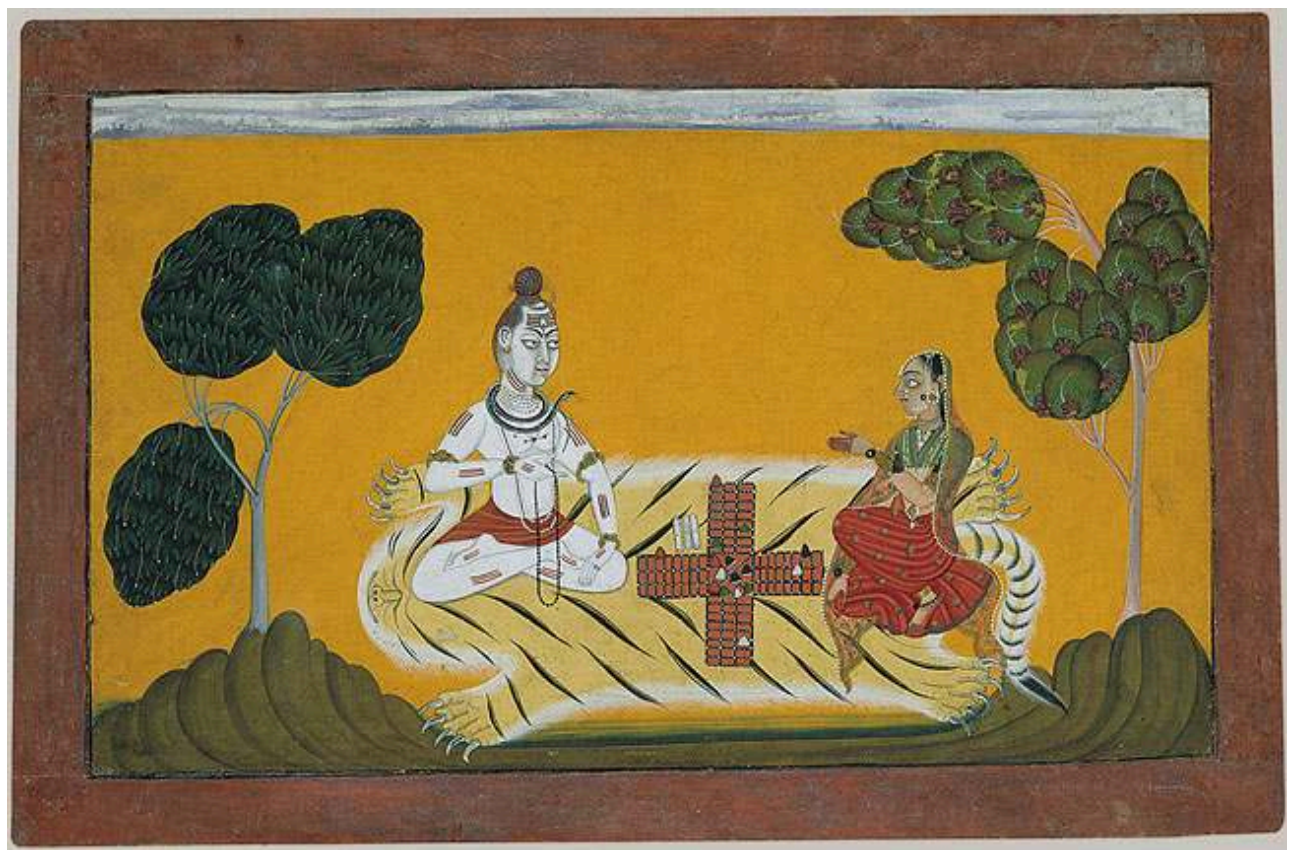

Devidasa of Nurpur, 1694-95, Metropolitan Museum of Art, New-York. 
Illustration 7 : Shiva et Parvati jouant aux dés

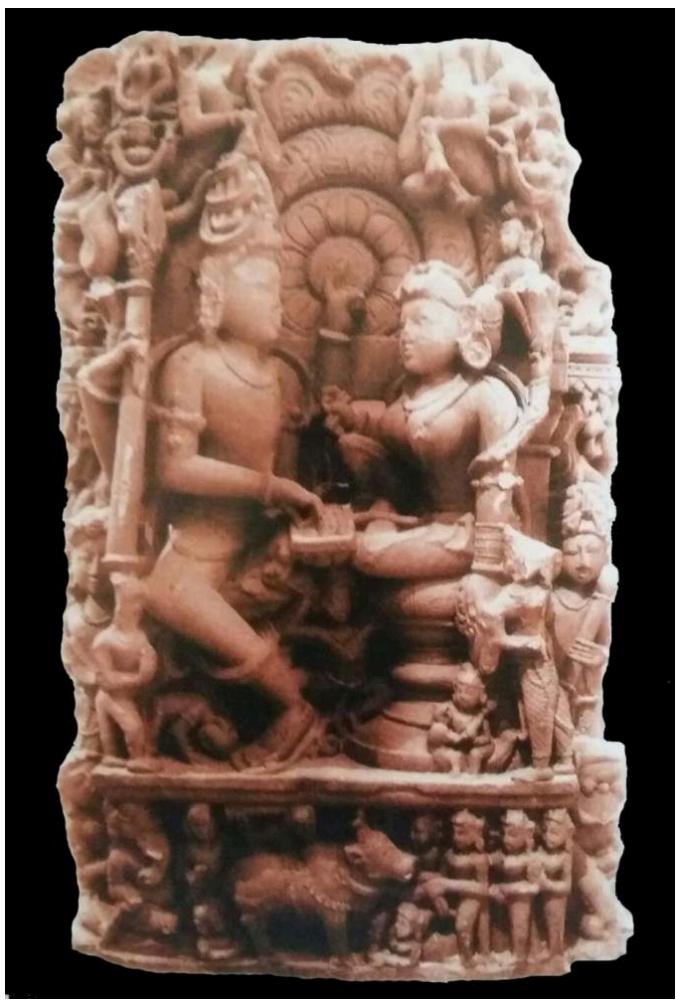

Statue de la dynastie Kalachuri, Xe siècle, Jabalpur Museum.

Illustration 8 : Relief des grottes d'Ellora montrant Shiva et Parvati jouant aux dés

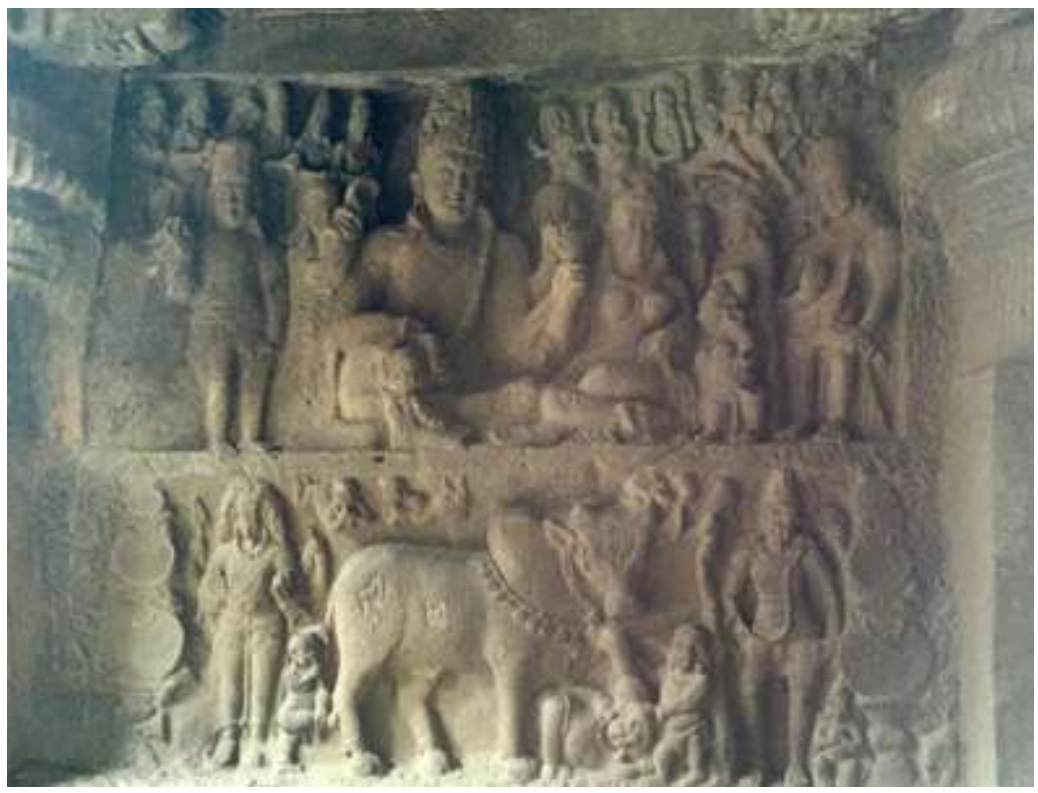

Grotte XXIX (Dumar Lena), Vle siècle. Photo: Sarah Welch, https://commons.wikimedia.org/wiki/ File:Shiva_and_Parvati,_Ellora.jpg

Gyan Chaupar et Pachisi se différencient en ce que le parcours, sur le premier, est entièrement déterminé par le jet du dé, alors que sur le second, il est également impacté par les actions des autres joueurs, et le joueur peut faire des choix, notamment 
quant à celle de ses pièces qu'il a intérêt à déplacer. Les deux jeux ont pu se développer à partir d'un même principe, celui d'un jeu de parcours déterminé par le jet de dés, mais se sont différenciés suivant deux principes différents: hasard pur et hasard associé aux décisions des joueurs.

En effet, le Pachisi est un jeu profane, dans lequel les joueurs peuvent faire des choix et interagissent. Il y a une possibilité, pour gagner aux Petits chevaux, de nuire à la progression des autres joueurs, possibilité qui est absente dans Serpents et échelles. Dans le Gyan Chaupar, le joueur ne fait que jeter les dés et parcourir les cases en prenant connaissance de leur contenu et de ce fait, le jeu se prête à des fins d'éducation morale et religieuse, voire à un usage divinatoire.

Dans les versions les plus anciennes du jeu en Inde et au Népal, qu'elles soient jaïn, hindoue ou bouddhiste, les lignes du plateau représentent les différents niveaux possibles d'existence de l'âme, en partant des plus matérielles et sommaires en bas, jusqu'aux plus spirituelles et à la libération (moksha) en haut. Selon les versions, les cases représentent des qualités, le joueur partant des instincts basiques pour évoluer, en passant par des appétits affectifs et sociaux, vers une recherche de plus en plus élevée. Des vertus particulières lui permettent de grimper par les échelles directement vers des états élevés, tandis que des vices, y compris cachés dans les états élevés, le font chuter à un niveau plus bas.

Le parcours représente ainsi l'évolution spirituelle de l'individu et les conséquences de ses actes en termes de réincarnation, le jet de dés jouant le rôle du karma. Dans la version bouddhiste tibétaine, ce sont les lieux de réincarnation qui sont figurés par les cases du plateau et le jeu consiste à progresser à travers plusieurs vies successives vers la libération.

L'occupant britannique avait observé l'usage éducatif qui était fait du Gyan Chaupar par les enseignants indiens pour sensibiliser leurs élèves aux vices et aux vertus, ainsi qu'au combat entre l'ordre et les passions. Le jeu est introduit dans une version adaptée à l'Angleterre victorienne vers la fin du XIX ${ }^{\mathrm{e}}$ siècle. La société Jaques of London affirme sur son site que c'est John Jaques qui, le premier, crée le jeu en $1873^{3}$. Dans les années qui suivent, plusieurs jeux comparables sont édités, sous des dénominations parfois différentes. Les valeurs indiennes sont remplacées par celles qui expriment les cadres moraux de l'époque : les cases "Accomplissement ", "Grâce » et "Succès » sont ainsi accessibles par des échelles partant de "Épargne », «Pénitence » et «Travail», et les serpents des cases «Complaisance », « Désobéissance » et «Indolence » font rechuter vers « Maladie », « Déshonneur » et « Pauvreté » (illustration 9). 
Illustration 9 : Jeu de Serpents et échelles de fabrication anglaise

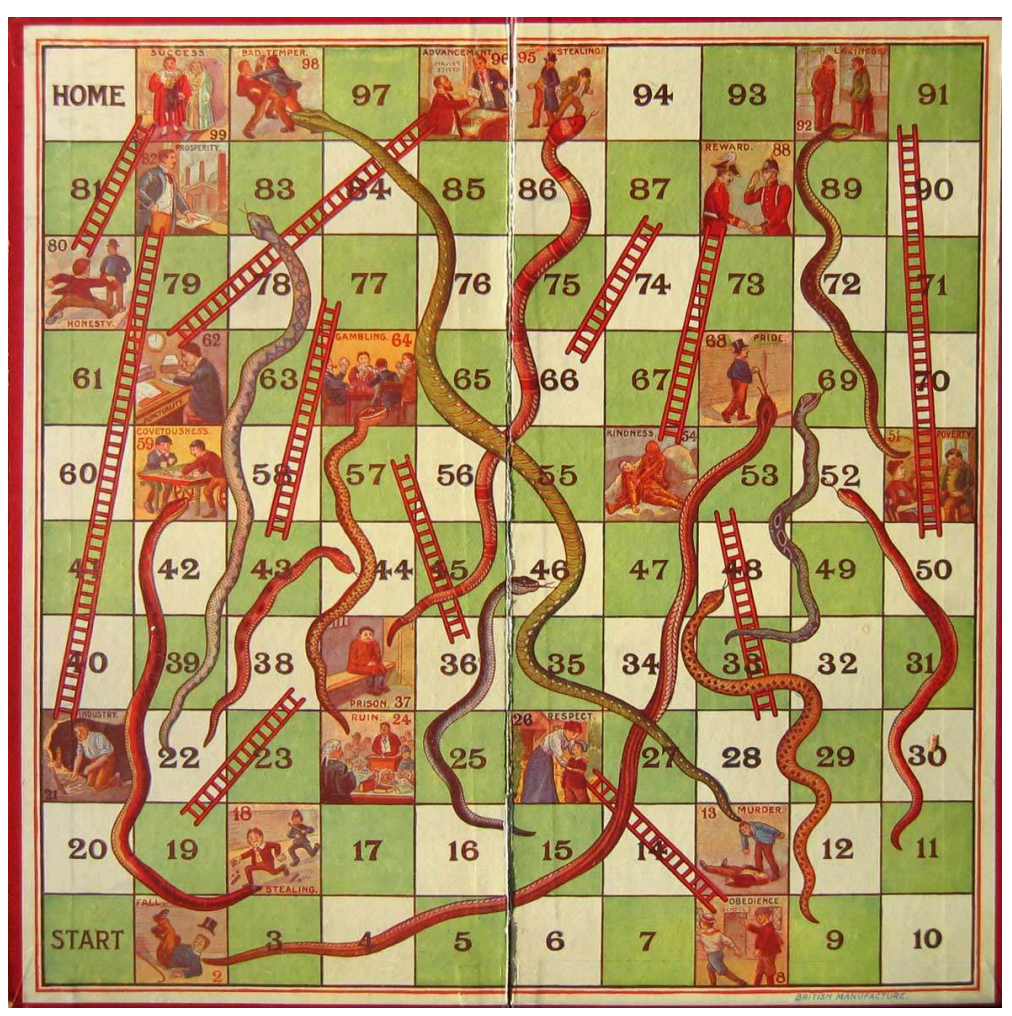

Papier imprimé collé sur carton, éditeur non précisé, début du XXe siècle.

21 Avec la décomposition de l'empire colonial et le retour des Britanniques au pays, les versions ultérieures du jeu tendent à se vider de leur contenu moral. En 1892, Frederick Henry Ayres, fabriquant de jeux concurrent de Jaques, dépose une licence pour un jeu de Serpents et échelles de cent cases en spirale, avec cinq serpents et cinq échelles, qui présente déjà des cases sans contenu (Finkel 2004). En 1943, le fabricant américain MB publie une version dépouillée, Chutes and Ladders ("Toboggans et échelles»), où les serpents sont remplacés par des toboggans et les contenus moraux se résument à des enfants accomplissant de bonnes actions en bas des échelles et récompensés au sommet, ou de mauvaises actions au départ des toboggans, avec leurs punitions en bas (illustration 10) (Augustyn 2004). 
Illustration 10 : Jeu de Chutes and Ladders (Toboggans et Échelles) de Milton Bradley

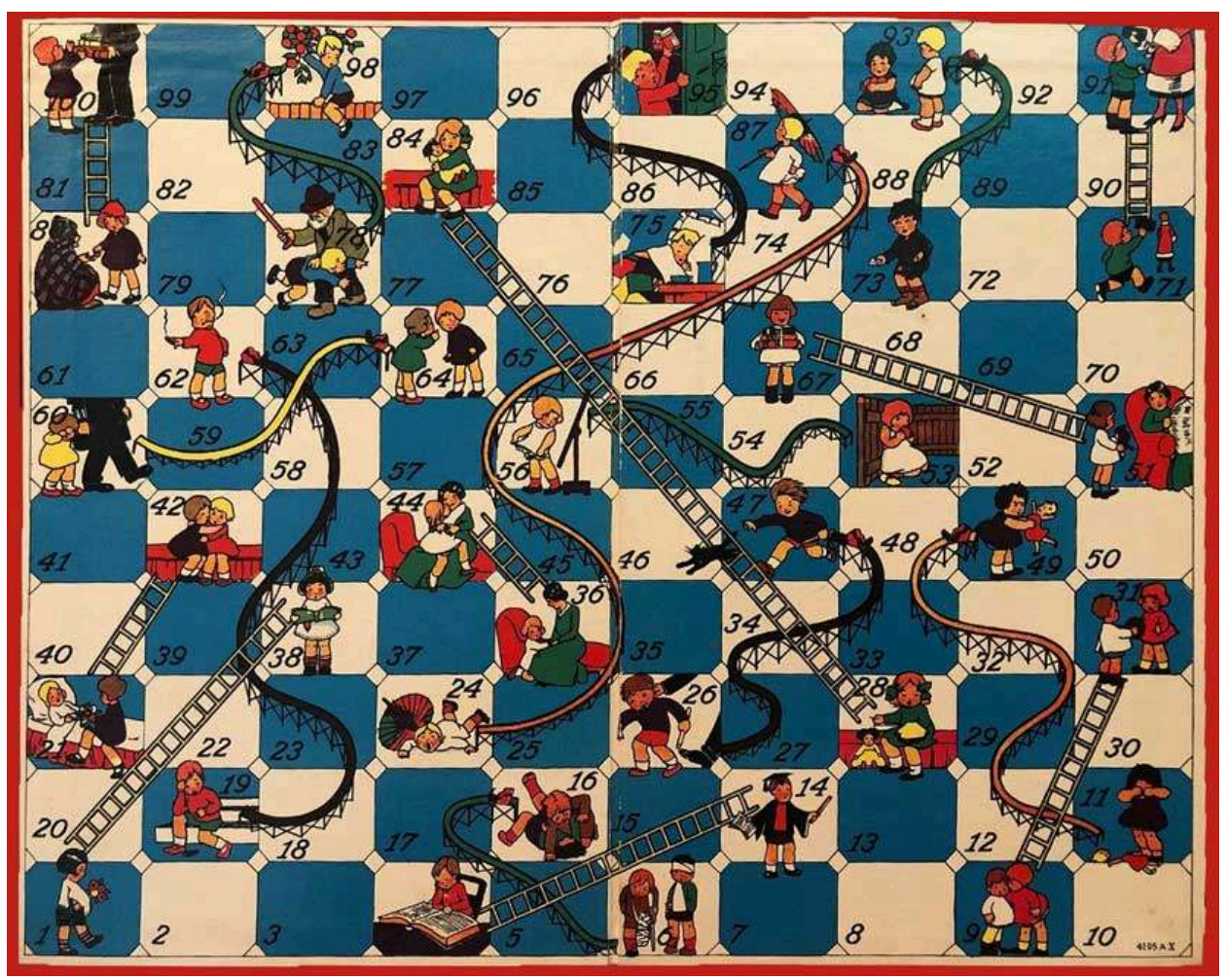

Papier imprimé collé sur carton, 1943.

22 Le jeu des Serpents et échelles tel qu'il est pratiqué en Occident de nos jours est dénué de tout contenu moral, religieux ou philosophique : à l'instar du Jeu de l'Oie ou du jeu des Petits chevaux, c'est un jeu de parcours qui fait intervenir le hasard du jet de dés, et dont l'enjeu est pour le joueur d'arriver le premier à la case terminale.

\section{Approche ludologique}

23 Qu'est-ce qui constitue l'essence de ce jeu? Existe-t-il une structure invariante, atemporelle et transculturelle, dont les diverses versions, à travers les époques et les aires culturelles, ne seraient que des déclinaisons adaptées à leur public? Auquel cas, les contenus narratifs des cases ne seraient qu'un habillage, et la version contemporaine des Serpents et échelles serait ce qu'il y a de plus proche de cette structure : une règle de déplacement sur un parcours.

24 Katie Salen et Eric Zimmerman (2003) rappellent qu'en analyse formelle, les règles d'un jeu constituent sa structure profonde, à partir de laquelle dérivent toutes les instances réelles du jeu. Pour illustrer la notion que, derrière les règles "opérationnelles" présentées au joueur, il existe des règles « constitutives » qui sont la structure formelle du jeu, ils donnent précisément l'exemple de Serpents et échelles. Ce jeu présente en effet une structure formelle assez simple qu'ils décrivent ainsi :

1. Tous les joueurs commencent avec une valeur zéro.

2. Les joueurs ajoutent chacun à leur tour un nombre aléatoire de 1 à 6 à leur valeur actuelle.

3. Le premier joueur qui atteint une valeur de 100 exactement gagne. Si l'ajout du nombre aléatoire au total du joueur excède 100 , on n'ajoute pas ce nombre ce tour-ci. 
4. Lorsque le total du joueur atteint certains nombres, le total change. Par exemple, si le joueur atteint 9 exactement, son total devient 31 ; si le joueur atteint 49 exactement, son total devient 11 ; etc. mouvements ne dépendent que du hasard et non de la décision du joueur, qu'ils sont indépendants des coups précédents et que les joueurs n'interagissent pas (Althoen et al., 1993). Dans la version à 100 cases et 19 toboggans et échelles, un joueur peut gagner en 7 coups, mais en moyenne il lui en faut 39,6. La structure du jeu implique que tirer le 6 ne permet nécessairement d'avancer le plus vite. Des jets de moindre valeur qui permettent d'utiliser les échelles et d'éviter les serpents permettent d'aller plus vite. À chaque case est ainsi associée une valeur optimale du jet de dé, celle qui permet d'aller le plus loin. De ce fait, par exemple, l'espérance du nombre de coups à jouer pour finir la partie est 9,71 si on se trouve sur la case 74 alors qu'elle est de 13,23 si on se trouve sur la case 81 qui est pourtant plus haut. En effet, en 74, on a plusieurs chances de prendre l'échelle de la case 80 , alors que cette échelle n'est plus accessible en 81 (Audet 2012). Serpents et échelles pour illustrer l'approche formelle d'un jeu en général est discutable, car il porte sur un jeu très particulier. La structure du Gyan Chaupar est simple, et sa description élégante, parce que la procédure est entièrement déterminée par le jet de dé. Elle exprime presque idéalement l'une des idées qui est au fondement de la théorie des jeux telle que la formulent John von Neumann et Oskar Morgenstern (1944, p. 49) : que « le jeu consiste dans l'ensemble des règles qui le décrivent ».

cette approche, les règles sont indépendantes des partenaires et de ce qu'ils peuvent éprouver : ce qui importe, c'est seulement quand un joueur peut choisir et quand il ne peut pas. Le joueur est un acteur sans intérieur, sans émotion, qui se résume à la somme des options dont il dispose à chaque coup et qu'il peut anticiper dans le but de conserver toujours au moins un degré de liberté (une possibilité de choix). Or, en l'espèce, le jeu propose un cas de figure où le joueur n'a aucun rôle à jouer, à part jeter les dés. Si ce qui importe, ce sont les options du joueur, dans ce cas, il n'en a pas d'autres qu'accepter ou refuser de jouer: sa seule liberté est de ne pas commencer la partie ou de l'interrompre en cours de route. Le jeu se joue à plusieurs, mais les actions des joueurs n'interfèrent pas entre elles: les parties de chacun se déroulent en parallèle. C'est en quelque sorte un jeu qui se joue sans joueur (le jet de dé pourrait être automatisé ou confié à un tiers), et on peut donc se demander s'il s'agit encore d'un jeu.

Dans cette version simple, le parcours n'offre pas de particularité ludique. Notons toutefois que les règles constitutives peuvent être enrichies, en favorisant par exemple certaines boucles de la chaîne de Markov, ou en affectant un parcours direct, non à une 
case, mais à un jet de dé, par exemple en obligeant le joueur à faire un six s'il veut quitter une case donnée, fonctionnant comme " prison ».

L'approche formelle ne permet pas de rendre compte de ce qui fait l'intérêt, pour le joueur, d'un type de jeu dans lequel il n'a pas de rôle à jouer, ni de degré de liberté comme il pourrait en avoir sur un plateau de dames ou d'échecs. S'il ne se passe rien dans les cases que traverse le joueur (i.e. s'il n'y a pas de narration à cet endroit), le design émotionnel du jeu est minimaliste: les émotions ressenties se réduisent à l'inquiétude précédant un jet de dé qui peut le faire tomber sur une «bonne » ou une « mauvaise » case, celle qui accélère sa progression ou celle qui le fait reculer.

\section{Approche narratologique}

L'intérêt du jeu, et son essence, seraient bien, non dans la structure formelle telle qu'elle s'exprime purement dans la version occidentale de Serpents et échelles, mais dans les contenus que l'on retrouve dans les formes traditionnelles du Gyan Chaupar.

Le jeu est un parcours à travers des situations décrites dans les cases. Chaque case comporte un numéro et représente selon les cas une qualité morale, une entité dans laquelle le joueur se réincarne, un état de développement spirituel ou un monde qu'il visite. On s'y arrête, on prend connaissance de ce que nous désigne la case, parfois on se réfère à un livret qui commente. Harish Johari (1993), qui propose un tel livret, affirme que par le passé, il fallait chanter un hymne correspondant à la case, mais que ces chants ont été perdus.

Le jeu est donc narratif, à l'instar de dispositifs comme les livres-jeux du type « Le livre dont vous êtes le héros » ou les jeux vidéo narratifs. Dans ces jeux, le joueur effectue des choix qui le conduisent à expérimenter des scénarios alternatifs. Mais son action se limite à ces choix qu'il fait entre plusieurs issues qui lui sont proposées, et il est ensuite observateur de ce qui se passe: il interagit avec la fiction, mais pas avec les personnages de celle-ci, et n'interfère pas dans l'action une fois qu'elle est déclenchée. Il est immergé dans l'histoire comme on peut l'être à l'écoute d'un conte ou au cinéma. Gyan Chaupar évoque ces fictions interactives, dans la mesure où, une fois le choix effectué, on assiste à ce qui se passe là où on est tombé. Certes, dans les fictions en question, le choix revient au joueur, alors qu'en l'espèce, c'est le sort qui décide. On peut alors restreindre la comparaison à des jeux vidéo contemplatifs comme Dear Esther (The Chinese Room, 2012) ou Gone Home (The Fullbright Company, 2013), où le joueur n'agit pas non plus et se laisse porter par le visuel, la musique, les textes de la fiction.

Le jeu est donc un dispositif narratif dans lequel le joueur est engagé à suivre les tableaux d'un récit dans un ordre aléatoire. Ce dispositif se prêterait à des contenus narratifs variés, dans des genres différents. Mais le contexte culturel indien a retenu des contenus religieux. Le rôle du jet de dés en tant que représentation d'un choix qui échappe au joueur n'est pas indifférent à cette destination. Le jeu sert les besoins d'une éducation aux valeurs morales, aux conséquences des bonnes et des mauvaises actions et attitudes, à l'essence de l'univers entre ordre et passion, karma et kama. C'est dans cet usage qu'il a été réceptionné dans l'Angleterre victorienne.

Bien entendu, cet usage "didactique", ainsi qu'il en est de tous les jeux sérieux, interroge l'essence proprement ludique du dispositif (game) et de son usage (play) : estce bien un jeu (Schmoll 2011)? L'une des caractéristiques définitoires d'un jeu est 
l'incertitude de son dénouement. Or, dans le Gyan Chaupar, comme dans une très grande proportion de jeux éducatifs, le parcours est pré-écrit, puisqu'il est dans l'objectif du dispositif de faire passer le joueur par un certain nombre de tableaux obligés pour qu'il «apprenne ». L'intérêt de la structure formelle de Serpents et échelles est de permettre statistiquement que le joueur passe et revienne sur une majorité de cases et en conséquence de tableaux. On peut cependant se demander si cet usage éducatif a été le seul en cours dans la tradition indienne. Jens Schlieter (2012) propose d'approcher le jeu comme une simulation. C'est-à-dire que le joueur ne fait pas qu'observer ou lire les contenus, pour " apprendre » les catégories de l'enseignement religieux, mais est invité à s'identifier à la situation de jeu pour faire l'expérience des actions décrites, en vivant les émotions. Il est en effet généralement demandé au joueur de prendre pour " pion » un objet personnel. Et nous ajouterons qu'après tout, même s'il s'agit d'un jeu de hasard pur, c'est néanmoins le joueur lui-même qui jette le dé, et non un tiers. Il est de la sorte impliqué par son incarnation dans le parcours qu'une loi extérieure, le jet de dé, lui imprime, l'obligeant à mourir et à renaître dans des états, selon les cas, plus bas ou plus élevés, jusqu'à ce qu'il connaisse la libération en atteignant la case terminale.

37 Jens Schlieter argumente à partir du jeu décrit par Mark Tatz et Jody Kent (1977) : il s'agit du jeu tibétain/népalais Sa Gnon Rnam Bzhags (« Monter les niveaux [spirituels]»), une version à 104 cases qui explore les différentes voies vers la libération (moksha) envisagées par le bouddhisme du « Grand Véhicule » (Mahayana) (illustration 11).

Illustration 11 : Version moderne du Sa Gnon Rnam Bzhags à 104 cases

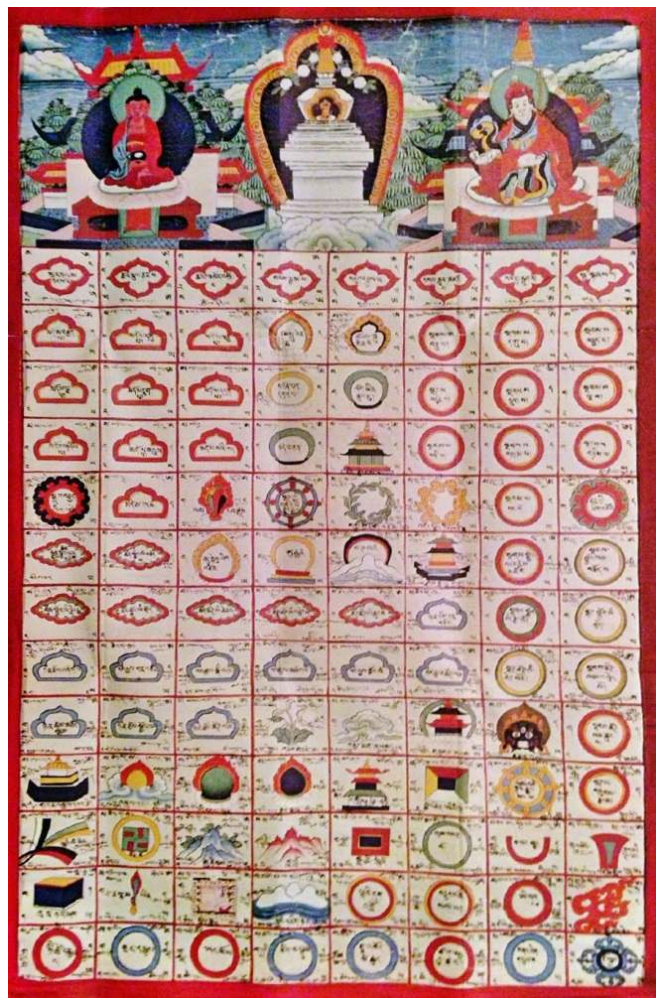

Peinture sur toile, 47 x 50 cm, 1971, anonyme reproduit in Tatz \& Kent (1977).

L'intérêt de cette version est qu'elle associe les contenus narratifs à une modification originale des règles procédurales. Certaines cases, associées à des échelles, favorisent certains trajets quand on s'y engage, tandis que d'autres capturent le joueur dans des 
boucles de la chaîne de Markov dont il a du mal, statistiquement, à sortir. Le dispositif narratif récupère donc à ses fins les propriétés du dispositif ludologique.

Le but du jeu est manifestement de démontrer que la libération ne peut être atteinte que par les deux voies du bouddhisme Mahayana, le bodhisattva-yana ( chemin des bodhisattvas", voie progressive qui consiste à passer par tous les degrés de la perfection) et le vajra-yana ("chemin de diamant», plus connu chez nous sous la désignation de "voie tantrique»). Toutes les autres voies, y compris celle du bouddhisme Hinayana, débouchent, dans la chaîne de Markov, sur des impasses.

Les cases figurent différentes représentations: paysages cosmologiques et lieux mythiques, étapes des voies de libération, certains dieux, d'autres traditions religieuses et des états de méditation. La case à atteindre est la 104. La pire est un enfer en bas à droite, qui est un piège dont on ne peut sortir qu'en tirant tous les nombres du dé autant de fois que la valeur correspondant à chaque face : une fois le 1, deux fois le 2, et ainsi de suite, jusqu'à six fois le 6 . Autant dire que celui qui tombe dans cet enfer a toutes les chances d'y rester coincé pendant le reste de la partie.

Une fois incarné dans l'une des six possibilités qu'offre la "grande route céleste » (case départ $n^{\circ} 24$ ), que l'on soit humain, animal ou démon, il s'agit, à chaque tour de jeu, de se réincarner dans une situation spirituellement plus élevée. Plusieurs voies sont possibles : les trois écoles du bouddhisme, Hinayana, Mahayana et tantrique, mais aussi d'autres religions. Seuls le Mahayana "classique» (le chemin des bodhisattvas) et la voie tantrique offrent des trajets qui mènent à la libération.

La voie tantrique est la plus rapide, mais c'est aussi la plus périlleuse (illustration 12). Incarné en humain, on peut sauter directement de la case 25 à la 72, où l'on maîtrise les huit siddhis (l'équivalent indien de "super-pouvoirs »). Mais si, à la case 33 (recherche de l'accumulation) qui fait partie de ce chemin, on tire un 6, on descend directement dans l'enfer vajra (case $n^{\circ} 1$ ). Cette chute est réservée au tantriste puisqu'elle n'est accessible que depuis la case 33 : elle sanctionne la trahison des vœux tantriques. Pour en sortir, on doit faire le nombre requis de jets, et il est alors possible que l'on tombe sur la case $n^{\circ} 9$ (Yama, seigneur de la mort), et de là, avec $50 \%$ de chance, on est directement de retour à une case élevée de la voie tantrique. Mais on peut aussi en sortir, via un champ de bouddhas, vers la voie progressive des Sutras. De la 33, on peut de même tomber sur le démon Rudra (case $n^{\circ} 16$ ), que l'on ne peut quitter qu'en tirant un 2, et de là, risquer de repasser par la voie tantrique. "Tantrique un jour, tantrique toujours ", pour paraphraser Schlieter : le chemin est rapide, mais un faux pas expose le pratiquant à rester prisonnier de ses pièges, car le tantrisme est une école dangereuse qui joue avec les passions. 


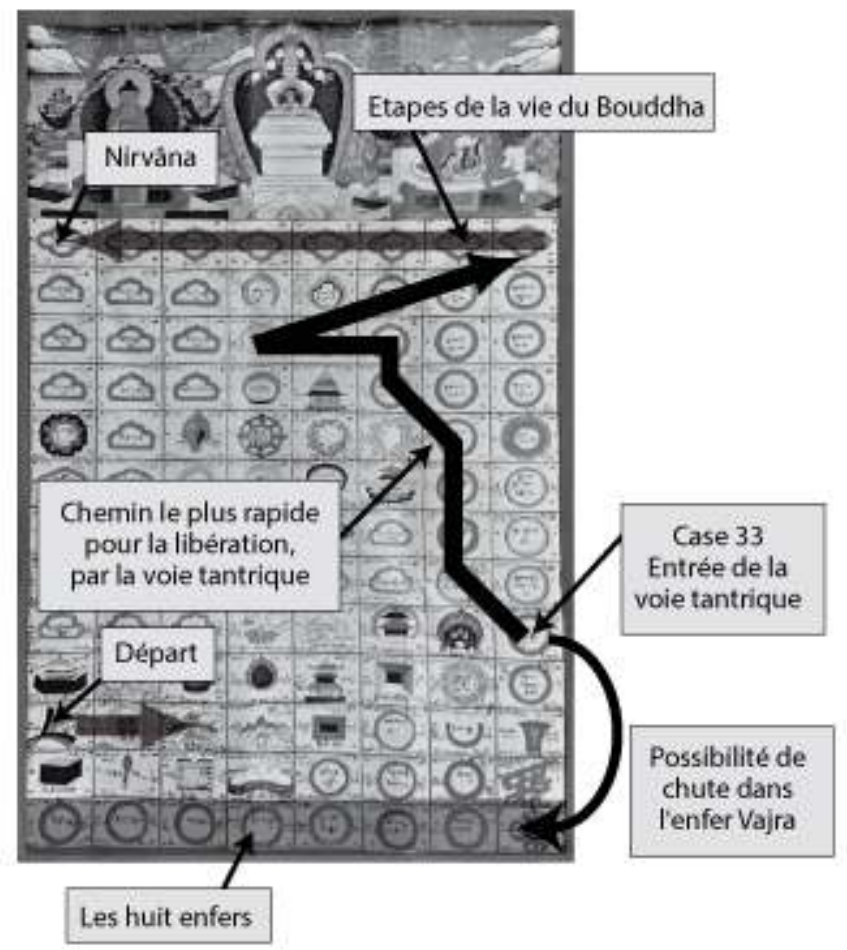

La voie des Sutras est plus sûre, car elle permet de ne pas tomber dans des pièges. Mais elle est aussi plus lente, image de l'élévation progressive par la lecture des textes. Seules les deux dernières cases de ce chemin permettent de passer à la case $n^{\circ} 84$, d'où il faut traverser tous les degrés de la perfection : toutes les cases de la ligne consacrée aux bodhisattvas, les unes après les autres (à chaque tour on avance d'une case sans jeter le dé).

comprend que le contenu narratif, accentué par les particularités d'un dispositif formel particulièrement accidenté, conforte l'hypothèse de schlieter que le jeu recherche ici l'identification et la simulation, pas seulement la prise de connaissance des contenus. L'immersion est plus poussée, et si la finalité est éducative, l'apprentissage est renforcé par l'expérience. À la différence de jeux vidéo, de films ou de récits, la narration, nous est cependant connue d'avance puisque son intégralité nous apparaît de façon synoptique sur le plateau avant que notre pion les traverse. On reste ainsi en présence d'un parcours, peut-être émotionnellement plus intense que sur d'autres Chaupar, mais qui laisse peu de surprises. Il nous reste donc à débattre de l'essence ludique, tant du dispositif que de ses usages : est-ce un jeu ? Question qui, par rebond, nous amène préalablement à en poser une autre : qu'est-ce qu'un jeu?

\section{Un monde de l'entre-deux : qu'est-ce qu'un jeu?}

L'une des caractéristiques du jeu les plus communément admises par les auteurs canoniques est son irréalité. C'est une action libre ressentie comme fictive et située en dehors de la vie courante (Huizinga, 1951), une activité accompagnée d'une conscience spécifique de réalité seconde ou de franche irréalité par rapport à la vie courante (Caillois, 1958). Le joueur y fait l'expérience du second degré, du faire-semblant, du 
«c'est pour de faux » (Brougère, 2005). Il résulte de cette caractéristique un rapport particulier de l'attitude ludique aux régimes de la connaissance, de la croyance et de la foi. Le jeu déconstruit la croyance, il est profanateur : on ne peut pas « jouer à croire». Enfiler une panoplie de prêtre pour jouer à faire la messe est antinomique du rite qui est ainsi imité, voire blasphématoire, car un «vrai » prêtre ne peut pas «faire semblant» de croire à ce qu'il fait. En cela, l'esprit de jeu est contraire à l'esprit religieux, le jeu est le contraire du sacré, le contraire d'un rituel (Caillois, 1958; Henriot, 1969; Schmoll, 2013 ; Di Filippo \& Schmoll, 2013 ; Renfrew, Morley et Boyd, 2018).

Cette différence, toutefois, exprime les catégories qui organisent notre univers romano-chrétien : on ne plaisante pas avec le sacré, par définition. Le monde réel est sérieux, et le jeu est le domaine, limité dans l'espace et le temps, des enfants et des dilettantes. Peut-être les Indiens partagent-ils une attitude différente, plus quotidiennement ludique, qui leur permet de réguler la coexistence au sein d'une société peuplée et cloisonnée par les castes et les divergences religieuses (Van Langendonckt, 2013) ? Il faut en tout cas rapporter cette caractéristique fictionnelle du jeu à la conception que la culture indienne se fait de la réalité.

Quatre concepts fondamentaux et solidaires nous introduisent au cœur des différentes philosophies indiennes : le karma, la mâyâ, la moksha et le yoga (Eliade 1954) :

1. Une loi de causalité universelle oblige l'homme, tout en se croyant libre, à accomplir des actes qui l'emprisonnent dans leurs conséquences, non seulement dans cette vie, mais dans les vies ultérieures qu'il est obligé de vivre pour les assumer : c'est le karma.

2. L'homme se croit libre parce qu'un processus l'entretient dans la nescience de la vérité : c'est la mâyâ, l'illusion de la réalité du monde, qui est dans l'hindouisme un pouvoir de Vishnu. La divinité entretient en l'humain la croyance que les objets de nos désirs sont consistants et qu'ils vont le satisfaire, alors que cette quête mène toujours à des frustrations et à la souffrance.

3. Il est possible d'accéder à la connaissance qui libère l'humain de cette illusion, de la souffrance et du cycle des renaissances : la moksha (libération) permet d'atteindre un état de non souffrance, d'infini, d'éternité. Dans le bouddhisme, cette libération est désignée par le terme de nirvana.

4. L'ensemble des moyens qui permettent d'atteindre la libération constitue les différentes formes de yoga. Ce terme ne désigne pas que les exercices posturaux et respiratoires dont nous avons l'image en Occident, mais toute méthode permettant d'arriver à ce résultat.

Les textes traditionnels indiens fourmillent d'histoires qui mettent en scène cette conception d'une réalité qui n'est qu'illusion, où les chercheurs de vérité supplient Vishnu de les faire accéder à la connaissance de sa mâyâ, ce à quoi la divinité répond en s'ingéniant malicieusement à leur faire vivre des aventures passionnelles douloureuses, dont ils découvrent, au final, qu'elles n'étaient qu'un rêve de quelques instants.

L'Inde a développé une vision du jeu comme paradigme de la transcendance divine (Colas, 1998). Deux conceptions du monde ont coexisté à cet égard. Dans l'une, l'illusion $\mathrm{du}$ monde manifesté ne recèle aucune transcendance: c'est, pour aller vite, la conception bouddhiste. Le monde ne nous apprend rien de ce qu'il y a au-delà de lui.

Dans une autre, plus positive, la mâyâ voile une réalité supérieure, mais elle est aussi la marque de l'existence de cet au-delà. Croire en la réalité du monde est une erreur, mais on peut apprendre de cette erreur. La création est alors considérée comme un jeu divin (lila ou leela). La divinité, Shiva ou Vishnu selon les écoles, illusionne les humains par sa 
magie. Les shivaïtes décrivent un dieu qui se mystifie lui-même jusqu'à oublier sa propre liberté de créateur. Les divertissements légendaires du dieu prennent valeur de mystères offerts à l'interprétation théologique et dévote. S'il est médité, le jeu ouvre sur la réalité vraie. Krishna est un avatar de Vishnu, joueur et séducteur, il attire par son chant les bouvières de la région, affolées d'amour pour lui. Il danse avec elles et les aime, puis disparaît, réapparaît et s'amuse. L'exégèse identifie les bouvières aux âmes en quête de Dieu et qui se perdent dans cette recherche. Le jeu, dans cette conception, n'est pas un divertissement: au contraire, il est une heuristique, enseigné par la divinité, qui permet de déconstruire l'illusion qui nous distrait de la vérité.

Dans les représentations, Shiva et Parvati jouent aux dés sur un plateau, et ils bluffent et trichent : c'est un vrai jeu. Mais les humains en sont les enjeux : le monde réel n'est que le plateau d'un jeu qui se joue sur une autre scène, transcendante. Cette conception du monde, on le voit, interroge la réalité en tant qu'apparence. Dès lors, si la réalité n'a pas plus de consistance qu'un jeu pour les dieux, existe-t-il un jeu qui en retour nous donnerait accès à la réalité qui est derrière l'illusion du monde?

Le plateau du Gyan Chaupar met en scène le samsara, le cycle des morts et renaissances auquel nous contraint le karma. Les Indiens qui jouent à Serpents et échelles sont dans leur élément, à ce jeu, un peu comme le Monopoly est en Occident une expression emblématique de la culture économiste dans laquelle nous vivons. Le joueur est engagé par l'incarnation dans le parcours qu'une loi extérieure, le jet de dés, lui imprime, l'obligeant à se réincarner dans différents états, selon les cas, plus bas ou plus élevés, jusqu'à ce qu'il connaisse la libération en atteignant la case terminale.

Dès lors, si le jeu permet de faire l'expérience simulée du cycle des morts et des renaissances, ainsi que des différentes voies possibles pour atteindre la libération, l'identification du joueur est-elle supposée lui permettre de mettre en jeu son propre karma, d'être informé de l'état où il se trouve présentement dans son propre cycle? Nous ne serions alors pas en présence d'un usage ludique ou ludo-éducatif, mais d'une pratique divinatoire ou initiatique.

Mark Tatz et Jody Kent (1977) estiment toutefois que le jeu n'était que rarement joué dans un but divinatoire, ce qui n'exclut pas qu'il ait pu l'être à l'occasion. Jens Schlieter ne pense pas non plus qu'il ait eu un tel usage. Dans les philosophies indiennes, argumente-t-il, le karma n'est jamais connaissable dans son influence sur le futur. Le jet de dé sur le plateau, même si l'on peut considérer qu'il est déterminé par son karma, ne devrait pas permettre de l'informer sur ce dernier. Schlieter ajoute que logiquement on pourrait rejouer immédiatement une nouvelle partie, avec un résultat probablement différent, et donc une interprétation également différente. Mais il admet que l'on ne sait pas si les pratiques prémodernes du Gyan Chaupar n'incluaient pas une interdiction de rejouer tout de suite, pour prévenir, justement, les contradictions entre deux interprétations oraculaires successives.

L'hypothèse de Schlieter est que le jeu est bien une simulation du karma du joueur, mais qu'elle n'est qu'une représentation approximative, incomplète, et pour tout dire fictionnelle, de la réalité. Le jeu, qui n'est qu'une fiction, une illusion consentie, exprime l'idée que le karma n'est lui-même qu'une fiction. Ainsi, tout est illusion. Le joueur est embarqué dans un jeu, fiction qu'il pense contrôler, puisqu'elle est consentie et que «ceci est un jeu ». Mais ce cadre est lui-même un trompe-l'œil, car il n'est pas exclu qu'il découvre, derrière le jeu, une vérité autre, ou que le jeu, en tant que fiction, 
"parle vrai», déconstruise ce que, chez nous, les auteurs baroques ont appelé le " grand théâtre du monde ".

La question de savoir si le Gyan Chaupar est un jeu s'expose en définitive à recevoir une réponse paradoxale, ou bien perd de sa pertinence. On pourrait dire, après Jacques Henriot $(1969,1989)$, que le jeu et la façon d'y jouer dépendent de ce troisième terme qu'est l'attitude ludique : la définition du dispositif comme plateau de jeu et comme activité ludique dépend de ce que le sujet a décidé, soit d'en faire un jeu et d'y jouer, soit de le consulter comme oracle ou de le pratiquer comme une forme non dite de yoga de la connaissance (jnana yoga). Mais on entrevoit que cela va un peu plus loin, car cette attitude ludique n'est pas toujours sous contrôle et sous consentement: que le joueur qui croyait avoir choisi de jouer découvre qu'il ne joue pas, mais qu'il est joué...

\section{Le statut du hasard : qu'est-ce que jouer?}

Comme ses ancêtres indiens, Serpents et échelles est un jeu de hasard, et plus précisément l'un des quelques jeux de hasard " pur », en ce sens que le déroulement de la partie est non seulement soumis au hasard, mais qu'il l'est totalement, à la différence des jeux de hasard dits raisonnés, qui sont lancés sur un processus aléatoire, mais dans lesquels les joueurs ont le choix entre plusieurs réactions possibles, par exemple (pour reprendre le cas des jeux de plateau comme le Pachisi), des options multiples de déplacement ou d'engagement de leurs pièces.

On doit se demander pourquoi l'enrichissement des règles, à travers les siècles, n'a jamais inclus, dans aucune des versions, la possibilité d'un choix de la part du joueur. Dans l'exemple de la version tibétaine, on pourrait imaginer que le joueur puisse choisir sa voie (bodhisattva ou tantrique) et subir les conséquences de ce choix, ce qui ferait sens : après tout, on met en scène le karma, et si le joueur choisit, il est confronté aux effets de ses actes. Ce n'est pas une licence que se sont donnés, à aucun moment, les concepteurs des diverses versions du Chaupar. Quel est le sens de maintenir tout le parcours sous l'empire du jet de dés? On doit supposer que le hasard remplit dans le jeu un rôle spécifique, lié à son statut dans le contexte culturel du jeu.

Dans toutes les sociétés prémodernes, qui admettent l'idée d'un au-delà du monde, le hasard a un statut transcendant. Quand César, décidant de franchir le Rubicon, lance son "Alea jacta est ", alea désigne certes en latin le jeu de dés : le dé est jeté. Mais le général romain ne veut pas dire par là que le succès de son entreprise est livré au hasard au sens où nous l'entendons, de processus aléatoire, mais bien qu'il fait un pari sur son succès parce qu'il croit en sa chance, en son destin, et/ou au soutien des dieux. Et quelque chose persiste de cette croyance aujourd'hui, chez les joueurs excessifs de jeux de hasard, qui les enferme dans la conviction qu'il y a un ordre des choses au-delà du raisonnable, ordre invisible mais dont ils ont l'intuition: cette intuition et leur ténacité, malgré les revers, sont les qualités qui justifieront - une fois qu'ils auront gagné, bien sûr - qu'ils étaient des élus.

A contrario, même les sociétés qui attribuent une transcendance au hasard reconnaissent les joueurs excessifs. Nous avons évoqué l'hymne 10-34 du Rig-Veda. De même, le Mahâbharata condamne les jeux de dés comme perte de temps et activité immorale (Topsfield, 2006a). Cet excès s'assimile selon les cas à une possession démoniaque ou à une volonté humaine insolente de forcer les dieux ou puissances du destin, ce dont les joueurs sont punis en retour. Le futur Bouddha, le jeune Siddhârta 
Gautama, passe pour avoir joué aux jeux au point d'inquiéter son entourage, qui le voyait se désintéresser des affaires politiques. Mais le jugement est pris dans une conception qui inscrit la question du hasard dans la nature du karma. Si notre destin est écrit, alors le jet de dés et son résultat expriment le karma du joueur.

Le chroniqueur et essayiste indien Devdutt Pattanaik (2007) propose une interprétation intéressante de ce qu'ont pu être les débats sur le hasard dans l'Inde ancienne et en quoi ils ont défini la distinction entre le Gyan Chaupar et le Pachisi. Gyan Chaupar met en jeu l'antagonisme entre le destin (karma) et les passions (kama), en insistant sur le destin, alors que Pachisi, qui combine le jet de dés et les décisions des joueurs, met l'accent sur la vie comme associant chance et libre arbitre. La coexistence des deux types de jeu témoignerait que les Indiens n'ont pas accepté sans protester une conception du karma comme destin inévitable : ils pensent que l'on peut forcer le futur, sinon ils ne feraient pas appel à la prière, à la divination ou à l'astrologie, mais seraient entièrement fatalistes. Le Pachisi exprime cette tendance en réaction à celle qu'exprime le Gyan Chaupar: le jet de dés dépend du destin, mais le mouvement des pièces dépend des choix des joueurs et de leurs capacités.

Le statut du hasard interroge la nature de jeu d'un exercice entièrement soumis au hasard. Gouverné tout du long par un processus aléatoire, Serpents et échelles ne sollicite pas les capacités du joueur, et si gagner par hasard ne veut rien dire, on ne voit pas trop ce qui constitue l'enjeu. On pourrait même supposer que le Pachisi existe et s'est développé comme jeu (de divertissement profane) parce que le Gyan Chaupar ne permettait pas cet usage ludique. Autrement dit: parce que le Gyan Chaupar, entièrement soumis au hasard, n'est pas un jeu.

Dans les catégories de Caillois (1958), l'alea entretient un lien étroit avec le sacré. Pour cet auteur, la parenté des jeux de hasard avec les pratiques divinatoires est manifeste. Alors que les jeux d'agôn renforcent la conscience de soi dans l'opposition à l'autre, dans les jeux d'alea, au contraire, le sujet s'abandonne au destin. Caillois remarque en particulier que les jeux de hasard sont les jeux humains par excellence: les mammifères supérieurs connaissent le jeu, entre eux et avec les humains, et on peut identifier chez eux des jeux de compétition, de simulacre et de vertige; en revanche, les animaux ignorent les jeux de hasard, car ils «ne sauraient imaginer une puissance abstraite et insensible, au verdict de laquelle ils se soumettraient d'avance par jeu et sans réagir »(Caillois 1958, p.37-38). Formulé autrement, seuls les jeux humains peuvent mettre en scène une intervention transcendante.

Pour aller dans ce sens, on peut souligner que l'alea auquel le joueur consent librement est de nature à susciter une expérience subjective particulière, dont les joueurs excessifs peuvent témoigner, mais qui est certainement amorcée de façon subliminale dans tout jeu de hasard (et plus généralement dans toute pratique faisant intervenir le hasard). Ce type de jeu implique un abandon du sujet à un fonctionnement qui lui échappe. Et paradoxalement, il en résulte un état d'indifférence et de liberté : quand il jette le dé, il n'est responsable de rien, n'a rien à anticiper, il se laisse aller à être l'observateur de ce qui lui arrive.

Nous avons relevé plus haut que dans le Chaupar, le dénouement de la narration était d'une certaine façon éventé par le plateau de jeu, qui offre au regard tous les avenirs possibles du joueur: on sait où on va, et on sait ce qu'on peut être appelé à traverser pour y aller. Le hasard met cet aspect en relief. Subjectivement, le joueur suit un trajet par certaines cases, trajet qui en écarte d'autres : le trajet l'inscrit dans le temps, dans 
la durée vécue subjectivement, il ne sait pas où il sera au prochain coup. Tandis que la vision synoptique du plateau est une vision hors du monde et hors du temps, où le passé, le présent et le futur sont simultanés. C'est donc le jet de dés, l'engagement du joueur, qui actionne l'inscription dans la durée. Mais le joueur constate en même temps la qualité artificielle de ce vécu du temps, puisqu'il n'est qu'un parcours linéaire parmi d'autres au sein de l'ensemble des parcours possibles dont il a sous ses yeux le spectacle instantané et permanent. Dans un jeu comme le jeu d'échecs, où tous les déplacements résultent des décisions du joueur, le calcul renforce l'implication du joueur, referme sa vision sur un seul parcours, le meilleur possible. Dans le Chaupar, le joueur voit, de l'extérieur, tandis qu'il s'observe se déplacer, que le jeu n'est que cela : un plateau.

On comprend dès lors pourquoi le Gyan Chaupar, malgré de nombreuses versions, a conservé son caractère de jeu de hasard pur. On aurait pu concevoir une version intégrant des possibilités de choix par les joueurs, mais ceux-ci se seraient alors retrouvés dans un jeu où ils auraient eu des décisions à prendre, qui les auraient réinscrits dans la logique ordinaire des actions commandées par un calcul de moyens pour des objectifs. Ainsi, et d'une certaine façon contrairement à ce qu'infère le chroniqueur indien précité (Pattanaik, 2007), le Chaupar n'insiste pas tant sur la soumission au destin que sur la liberté que confère de s'en remettre à lui.

\section{Conclusion}

L'intérêt des lignes qui précèdent nous semble résider dans l'interrogation qu'elles devraient susciter sur «ce qu'est un jeu» et sur «ce qu'est jouer». Le concept « d'attitude ludique » devrait nous permettre de déterminer si quelqu'un est en train de jouer, en fonction de la disposition d'esprit dans laquelle il se trouve. Mais un sujet estil toujours certain de son attitude ludique? Quand on joue à pile ou face, est-ce que l'on sait toujours si l'on joue ou si l'on consulte un oracle ou une instance décisionnelle non humaine?

68 Cette question est rendue plus complexe quand on passe d'une culture à une autre. En fait, elle est peut-être seulement celle que se poserait un sujet occidental, imprégné de culture romano-chrétienne, pour qui il doit exister une frontière ferme entre le jeu et les affaires quotidiennes, entre la fiction et la réalité.

Pour un sujet indien, est-ce que le Gyan Chaupar est un jeu, une pratique divinatoire, une expérience simulée du samsara? Et surtout, la question est-elle pertinente, dans un environnement culturel qui prêterait plus volontiers à une lecture fractale du plateau comme miniature du monde réel... lequel n'est qu'une fiction en dernière instance. Ce que toutefois révèle le Chaupar, et même une pratique de Serpents et échelles dans sa version contemporaine dépouillée de contenus religieux, c'est un aspect qui pourrait être commun à nombre de jeux et de façons de jouer. Tout est illusion, et jusqu'au jeu lui-même: on pense ne faire "que» jouer, au sens d'une activité profane et de divertissement, et on finit par, "sérieusement ", apprendre quelque chose sur soi et sur l'essence du monde. 


\section{BIBLIOGRAPHIE}

ALTHOEN S.C., KING L. \& SCHILLING K. (1993). « How Long is a Game of Snakes and Ladders? », The Mathematical Gazette, 77, 478, pp. 71-76.

AUDET D. (2012), « Probabilités et espérances dans le jeu de serpents et échelles à deux joueurs », Bulletin de l'Association mathématique du Québec, LII, 4, pp. 28-40.

AUGUSTYN F.J. (2004), Dictionary of toys and games in American popular culture, New York NY, Haworth Press.

BORNET P. \& BURGER M. (eds) (2012), Religions in Play. Games, Rituals, and Virtual Worlds, Zurich, Pano Verlag.

BRODY A. (2015), « Pour une approche du gambling en termes de jeu », Sciences du jeu, 3, http:// journals.openedition.org/sdj/465.

BROUGÈRE G. (2005), Jouer/Apprendre, Paris, Economica.

CAILLOIS R. (1950), L'homme et le sacré, $2^{\mathrm{e}}$ édition, Paris, Gallimard.

CAILLOIS R. (1958), Les jeux et les hommes : Le masque et le vertige, Paris, Gallimard.

COLAS G. (1998), « Jeux humains, jeux divins. Vues indiennes », Extrême-Orient, Extrême-Occident, 20, pp. 157-163.

DI FILIPPO L. (2014), « Contextualiser les théories du jeu de Johan Huizinga et Roger Caillois », Questions de communication, 25.

http://journals.openedition.org/questionsdecommunication/9044.

DI FILIPPO L. \& SCHMOLL P. (2013), « Mise en scène et interrogation du sacré dans les jeux vidéo », Revue des sciences sociales, 49, pp. 64-73.

DUFLO C. (1997), Jouer et philosopher, Paris, P.U.F.

ELIADE M. (1954), Le yoga. Immortalité et liberté, Paris, Payot.

FINKEL I. (2004), «The ups and downs of life: the Indian game of snake and ladders », in C. Mackenzie \& I. Finkel (eds). Asian games: the art of contest, New York, Asia Society, pp. 58-63.

HAMAYON R. (2012), Jouer. Étude anthropologique à partir d'exemples sibériens, Paris, La Découverte.

HAWKING S. \& MLODINOW L. (2011), Y a-t-il un grand architecte dans l'univers ?, Paris, Odile Jacob.

HENRIOT J. (1969), Le jeu, Paris, PUF.

HENRIOT J. (1989), Sous couleur de jouer. La métaphore ludique, Paris, José Corti.

HUIZINGA J. (1951 [1938]), Homo ludens. Essai sur la fonction sociale du jeu, Paris, Gallimard.

JOHARI H. (1993), Leela, The Game of Self-Knowledge, Rochester VT, Destiny Books.

PATTANAIK D. (2007), « Playing with Fate and Free Will », First City magazine, New Delhi. http:// devdutt.com/articles/indian-mythology/playing-with-fate-and-free-will.html.

RENFREW C., MORLEY I., BOYD M. (eds) (2018), Ritual, play and belief, in evolution and early human societies, Cambridge, Cambridge University Press.

SALEN K. \& ZIMMERMAN E. (2003), Rules of Play. Game Design Fundamentals, Cambridge MA, The MIT Press. 
SCHLIETER J. (2012), « Simulating Liberation: The Thibetan Buddhist Game "Ascending the [Spiritual] Levels" ", in Ph. Bornet \& M. Burger (eds), Religions in Play. Games, Rituals, and Virtual Worlds, Zurich, Pano Verlag, pp. 93-116.

SCHMIDT-MADSEN J. (2013), Road Maps for the Soul. A critical reading of five North Indian 72-square gyān caupar boards, M.A. Specialized Topic Paper, University of Copenhagen.: http:// themathesontrust.org/papers/hinduism/Schmidt-Madsen-Road_Maps_for_the_Soul.pdf

SCHMIDT-MADSEN J. (2019), The game of knowledge: playing at spiritual liberation in 18th-and 19thcentury Western India., thèse de doctorat, Københavns Universitet, Det Humanistiske Fakultet.

SCHMOLL P. (2010), « Jeux sans fin et société ludique », in S. Craipeau, S. Genvo \& B. Simonnot (dirs), Les jeux vidéo au croisement du social, de l'art et de la culture, Metz, Questions de communication, pp. 27-42.

SCHMOLL P. (2011), « Jeux sérieux : exploration d'un oxymore », Revue des sciences sociales, 45, pp. 158-167.

SCHMOLL P. (2013), « Relire Jacques Henriot à l'ère de la société ludique et des jeux vidéo », Sciences du jeu, 1, https://journals.openedition.org/sdj/271.

SHIMKADA D. (1983), « A Preliminary Study of the Game of Karma in India, Nepal, and Tibet », Artibus Asiae, 44(4), pp. 308-322.

SOAR M. (2006), « Shiva and Parvati at play. Backgammon in ancient India ». in A. Topsfield (ed.), The art of play. Board and card games of India, Mumbai, Marg Publications, Quartely Books 58(2), pp. 44-59.

SOAR M. (2007), « Board games and backgammon in ancient Indian sculpture », in I.L. Finkel. (ed.), Ancient board games in perspective : papers from the 1990 British Museum colloquium, with additional contributions. London, The British Museum Press, pp. 177-231.

TATZ M. \& KENT J. (1977), Rebirth: The Tibetan Game of Liberation, New York NY, Anchor Press. TOPSFIELD A. (1985), « The Indian Game of Snakes and Ladders », Artibus Asiae, 46(3) pp. 203-226. TOPSFIELD A. (2006a), « Dice, Chaupar, Chess. Indian games in history, myth, poetry and art », in A. Topsfield (ed.), The art of play. Board and card games of India, Mumbai, Marg Publications, Quartely Books 58(2), pp. 11-31.

TOPSFIELD A. (2006b), « Snakes and Ladders in India: Some Further Discoveries », Artibus Asiae, 66(1), pp. 143-79.

VAN LANGENDONCKT M. (2013), « L'Inde, microcosme ludique ? », Colloque de l'association Asia Jeux indiens et originaires de l'Inde, Bruxelles, Haute école de Bruxelles-Defré \& Université Libre de Bruxelles, décembre. http://ludobel.be/wp-content/uploads/2015/12/

ColloquejeuxindiensVLG.pdf

VON NEUMANN J. \& MORGENSTERN O. (1944), Theory of games and economic behavior, Princeton NJ, Princeton University Press.

\section{NOTES}

1. La sotériologie est le domaine de la théologie qui traite des théories du salut de l'âme.

2. Mandala 10, hymne 34 : https://en.wikisource.org/wiki/The_Rig_Veda/Mandala_10/Hymn_34

3. https://www.jaqueslondon.co.uk/collections/snakes-and-ladders 


\section{RÉSUMÉS}

Serpents et échelles est la version profane, diffusée aujourd'hui en Occident, d'un jeu qui est pratiqué depuis des millénaires en Inde. Il soumet entièrement le joueur à la décision du jet de dés et est à ce titre un parangon des jeux de hasard. L'article présente les caractéristiques et l'histoire de ce jeu. Aussi bien ses contenus narratifs dans ses contextes culturels d'origine, que le principe même d'un jeu dans lequel le joueur ne décide de rien, permettent de se demander : estce un jeu? Cette question en amène une autre : qu'est-ce qu'un jeu? L'exploration des usages de Serpents et échelles dans ses formes anciennes révèle un aspect que dissimule la version contemporaine, dépouillée de ses contenus sotériologiques, mais dont elle pourrait conserver une part de l'efficacité : l'abandon aux décisions du hasard permet une expérience simulée de la transcendance.

Snakes and Ladders is the Western and secular version of a game that has been played for millennia in India. It completely submits the player to the decision of the dice, and is, as such, a paragon of games of chance. The article presents the characteristics and history of this game. Both its narrative contents in its original cultural contexts, as well as the very principle of a game in which the player decides nothing, allow us to ask: is it a game? This question leads to another: what is a game? The exploration of the uses of Snakes and Ladders in its old forms reveals an aspect concealed by the contemporary version, stripped of its soteriological content, but whose effectiveness could partly be retained: the abandonment to the decisions of chance allows a simulated experience of transcendence.

\section{INDEX}

Keywords : Snakes and ladders, Gyan Chaupar, games of chance, definitions of game, transcendence

Mots-clés : Serpents et échelles, Gyan Chaupar, jeux de hasard, définitions du jeu, transcendance

\section{AUTEURS}

\section{SERGE FINCK}

Studios Almédia \& Ernestine (Strasbourg)

PATRICK SCHMOLL

PSInstitut (Strasbourg)

UMR DynamE (Univ. Strasbourg/CNRS) 